\title{
Exploring Reversible Universal Gate with 13 Standard Function and Symmetric Function Implementation
}

\author{
Saroj Kumar Chandra \\ Assistant Professor, Department Of Computer Science \& Engineering, \\ Chouksey Engineering College, Bilaspur (Chhattisgarh), India,
}

\begin{abstract}
Quantum-dot Cellular Automata (QCA) is naval technology for development of logic circuits based on nanotechnology and it is an one of the alternative for designing high performance computing over existing CMOS technology. The basic logic in QCA does not use voltage level for logic representation rather it represent binary state by polarization of electrons on the Quantum Cell which is basic building block of QCA. Extensive work is going on QCA for circuit design due to low power consumption and regularity in the circuit. Reversible logic design is a well-known paradigm in digital computation, and in this paper we are presenting the effectiveness of Reversible Universal Gate (RUG) with realization of 13 standard function and symmetric functions using RUG.
\end{abstract}

\section{Keywords}

Reversible Universal Gate, Quantum Cell, Quantum Dot Cellular Automata.

\section{INTRODUCTION}

The current digital design techniques target energy efficient realization of complex logic circuits .The QCA (Quantum-dot Cellular Automata) is considered to be a promising technology to meet such a design target (device density of $10^{12}$ device $/ \mathrm{cm}^{2}$ ). It achieves switching speed of $10 \mathrm{ps}$ and power dissipation of the order of $100 \mathrm{~W} / \mathrm{cm}^{2}$ [1].

The fundamental operational unit of QCA based design is the 3 -input majority gate (majority voter). Since the majority gate is not functionally complete, the majority gate with inverter, called MI, and are used to realize the QCA designs. The universal gate structures such as AOI (and-or-inverter)[2] have been proposed to achieve the cost effective digital design. However, these gates realize the irreversible logic and, therefore, can't be the right choice for energy efficient design.

Landauer [3] proved that for irreversible logic computations, each bit of information loss generates $\mathrm{k}_{\mathrm{B}} \mathrm{T} \ln 2$ joules of heat energy as the energy required for a binary transition $\mathrm{E}_{\mathrm{bit}}$ is given by the Shannon-von Neumann- Landauer (SNL) expression [3]

$\mathrm{E}_{\mathrm{bit}} \geq \mathrm{E}_{\mathrm{SNL}}=\mathrm{k}_{\mathrm{B}} \operatorname{Tln} 2=0.017 \mathrm{eV},(1)$

Where $k_{B}$ is the Boltzmann constant, $T=300 \mathrm{~K}$. This is the minimum energy to process a bit. However, the reversible computation allows computing beyond the SNL limit. Bennett [4] showed that a zero power dissipation in logic circuits is possible only if a circuit is composed of reversible logic gates.
The reversible logic conserves energy using a charge recovery process in CMOS. On the other hand, the QCA circuit is a clocked information preserving system [5]. The energy dissipation of a QCA circuit can be significantly lower than $\mathrm{k}_{\mathrm{B}} \mathrm{T} \ln 2$. This energy conservation is extra feature in favor of the QCA based circuit design. However QCA is more error prone to circuit designation [2].

The above scenario leads to another approach to design new gate structure based on QCA that will behave in reversible manner and also realizes the universal logic function. QCA based reversible circuit design satisfies the requirement for energy saving and at the same time ensure the defect tolerance. The reversible universal logic gate (RUG) has already proposed [6] that reduce the number of logic gates and garbage outputs in a digital design around it as compared to other existed reversible gates. Reversible Universal gate (RUG) is area saving implementation of complex logic simultaneously ensuring energy loss close to zero. According to [6] shown that the effectiveness of any circuit can evaluated with implementation of 13 standard function and two and three variable symmetric function implementation so in this paper we are presenting the effectiveness of RUG with realization of 13 standard function and symmetric functions.

\section{The Reversible Logic}

A logic gate $\mathrm{L}$ is reversible if, for any output $\mathrm{Y}$, there is a unique input $\mathrm{X}$ such that

$$
\mathrm{L}(\mathrm{X})=\mathrm{Y}
$$

If a gate $\mathrm{L}$ is reversible, there is an inverse gate $\mathrm{L}^{\prime}$ which maps $\mathrm{Y}$ to $\mathrm{X}$ for which

$$
L^{\prime}(Y)=X .
$$

From common logic gates, NOT gate is reversible.

The original motivation was that reversible gates dissipate less heat (or, in principle, no heat). In a normal gate design, input states are lost after producing the output since less information is present in the output than was present at the input. This loss of information loses energy to the surrounding area as heat because of thermodynamic entropy. Another way to understand this is that charges on a circuit are grounded and thus flow away, taking a small charge of energy with them when they change state. A reversible gate only moves the states around and since no information is lost so energy is conserved. Any reversible gate must have the same number of input and output bit. The most studied reversible logic gates are the NOT, Toffoli, Fredkin gate, CNOT or Feynman, Peres etc. [7], [8] as shown in Fig.1. 


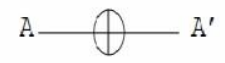

(a) NOT

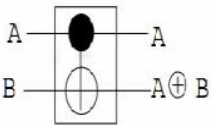

(b) C-NOT or Feynman gate

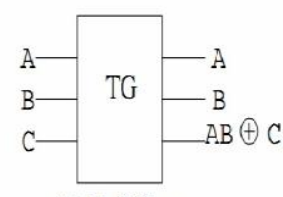

(c) Toffoli gate

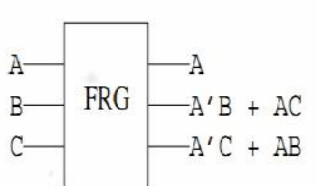

(d) Fredkin gate

\section{Reversible Universal Gate}

Characterization of thirteen variable standard functions [2] and symmetric functions [9] raises the following design issues:

- What are the best possible logic function(s) that can implement Boolean logic functions efficiently?

- How can the number of logic gates be minimized in a design to make it more cost-effective?

- How to ensure energy efficient computing avoiding the information loss in a circuit realizing complex logic?

All the above characteristic leads to design of new logic gate design and new gate reversible universal gate (RUG) proposed [6] in which we found all the properties mentioned above. The RUG is three inputs and three outputs logic design as shown in Fig.2.

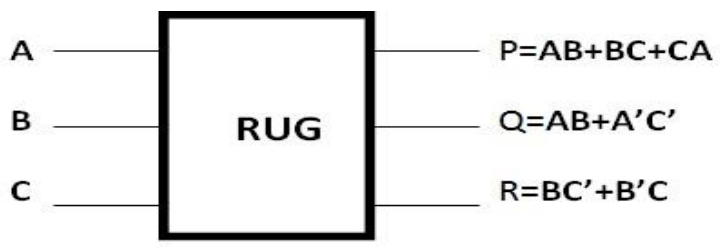

FIG.2: Reversible Universal Gates

The $\mathrm{Q}=\mathrm{AB}+\mathrm{A}^{\prime} \mathrm{C}^{\prime}$ is called Universal Function and it is a function which can achieve any logic with a given number of variables with minimum number of wire crossings [10]. So from [10], presence of universal function RUG based QCA circuits are very much cost-effective in terms of number of logic gate, wire crossing, cells count as compared to the designs based on other existing reversible gate. This is the concept of a special form of reversible logic with universal functionality. So the effectiveness of RUG in digital design is evaluated in realizing the 13 standard functions and symmetric functions.

\section{Realizing 13 standard function}

The thirteen standard functions (shown in Table I) represent all 256 3-variable Boolean functions, since any 3-variable Boolean function can be converted to one of the thirteen standard functions [2]. The following table shows realization of all thirteen function using RUG gate.
Table I: Implementation of All 13 Standard Function Using RUG

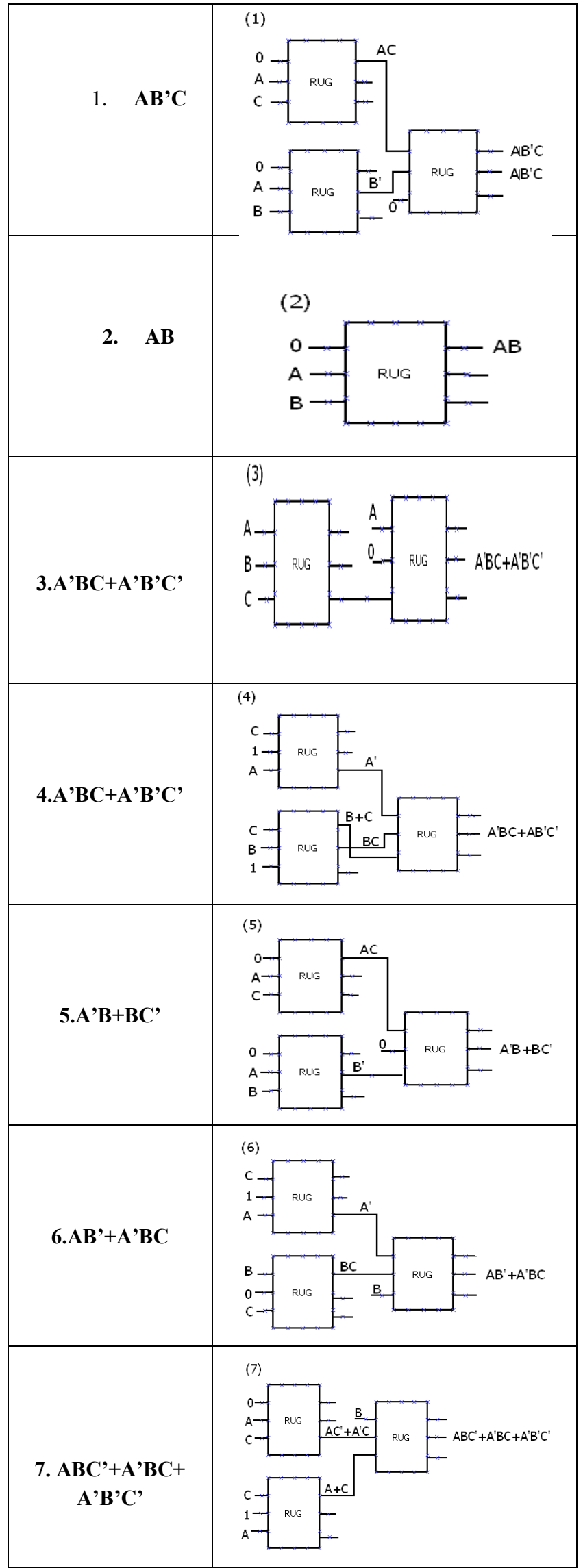




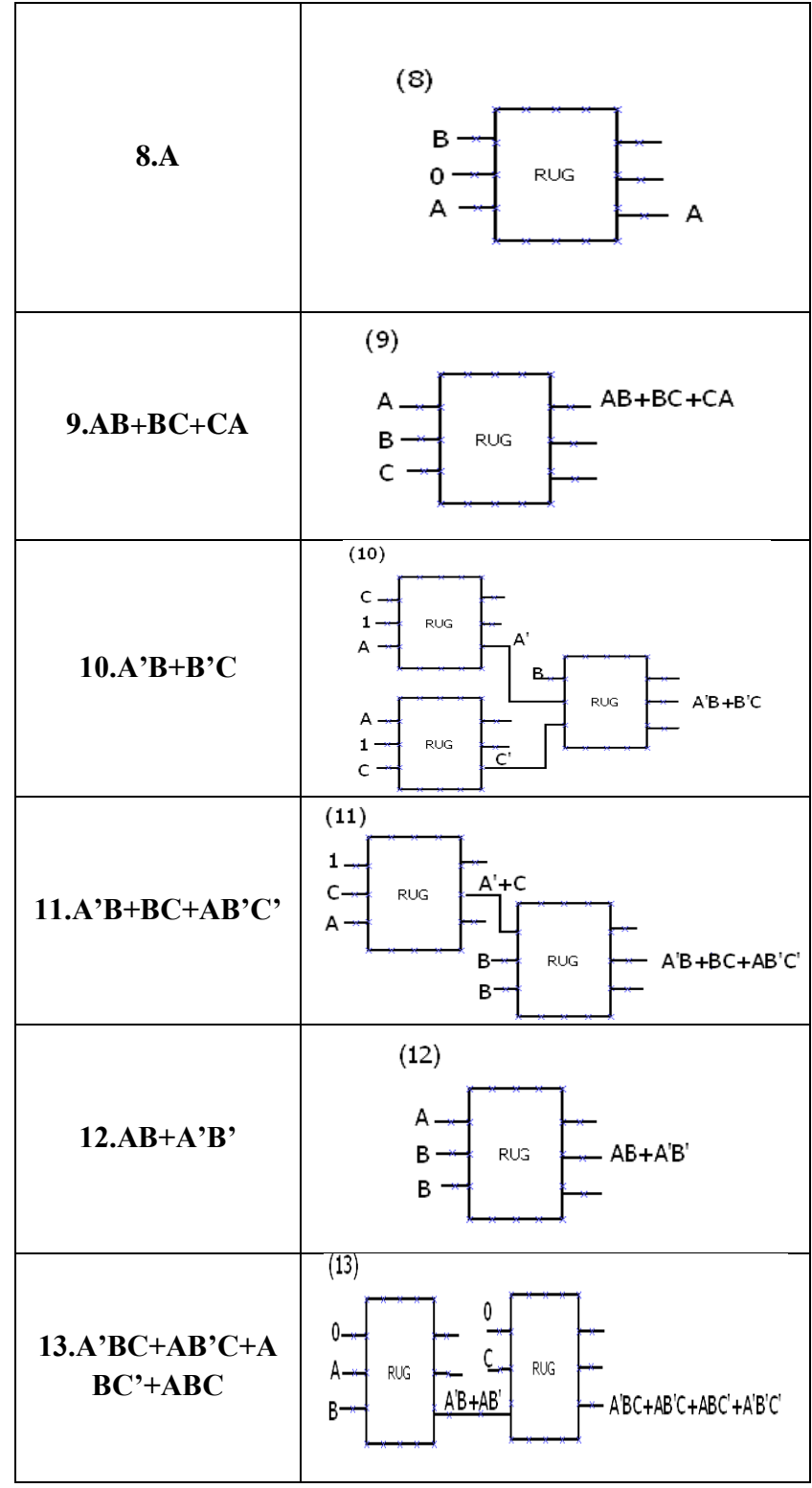

The comparison graph among various reversible gates for implementation of 13 standard functions is given in the Fig.3.

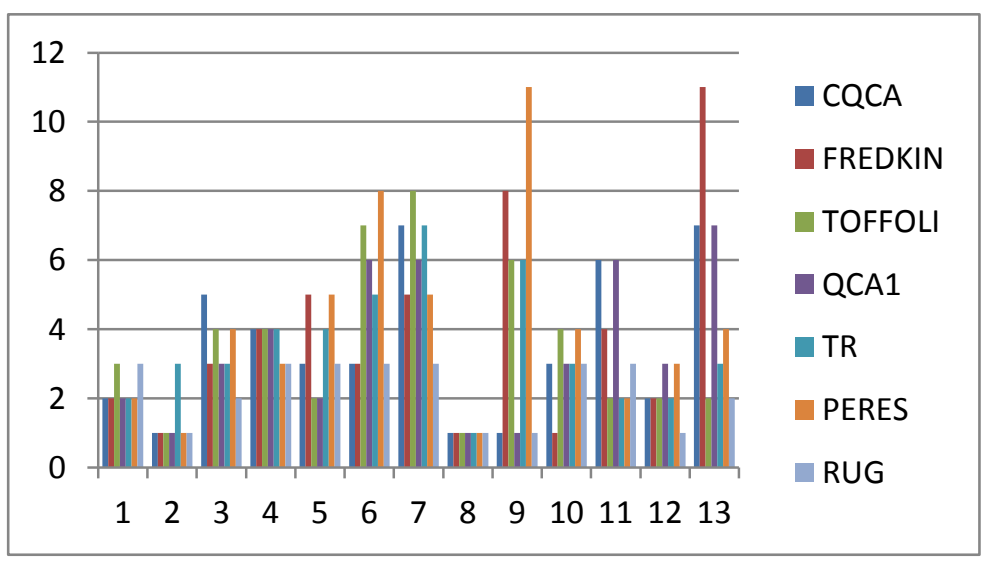

FIG.3: Number of gates taken to implement 13 standard function by different reversible gates(y coordinate) vs. 13 standard function. ( $x$ coordinate)

\section{Two and Three Variable Symmetric Function Implementation by RUG}

A switching function $\mathrm{f}(\mathrm{x} 1, \mathrm{x} 2 \ldots \mathrm{xn})$ is called symmetric (or totally symmetric) with respect to the variables $\mathrm{x} 1, \mathrm{x} 2 \ldots \mathrm{xn}$, if it is invariant under any permutation of its variables. The symmetric functions are receiving consider able attention from the researchers working in the field of VLSI design [9]. A number of synthesis techniques for boolean symmetric functions are reported [9] to suit different applications.

Table II: three variables Symmetric Function

\begin{tabular}{|c|c|}
\hline Q1 & $\mathrm{XYZ}$ \\
\hline Q2 & $\mathrm{X}+\mathrm{Y}+\mathrm{Z}$ \\
\hline Q3 & $\mathrm{X}^{\prime}+\mathrm{Y}^{\prime}+\mathrm{Z}^{\prime}$ \\
\hline Q4 & $X^{\prime} Y^{\prime} Z^{\prime}$ \\
\hline Q5 & $\mathrm{XY}+\mathrm{YZ}+\mathrm{ZX}$ \\
\hline Q6 & $\mathrm{X}^{\prime} \mathrm{Y}^{\prime}+\mathrm{Y}^{\prime} Z^{\prime}+\mathrm{Z}^{\prime} \mathrm{X}^{\prime}$ \\
\hline Q7 & $\mathrm{X}^{\prime} \mathrm{Y}^{\prime}+\mathrm{Y}^{\prime} \mathrm{Z}+\mathrm{Z}^{\prime} \mathrm{X}+\mathrm{ZX} \mathrm{X}^{\prime}+\mathrm{XY} \mathrm{Y}^{\prime}+\mathrm{Y}^{\prime}$ \\
\hline Q8 & $\mathrm{XYZ+X^{ \prime } Y ^ { \prime } Z ^ { \prime }}$ \\
\hline Q9 & $\mathrm{XYZ}^{\prime}+\mathrm{XY} \mathrm{Z}^{\prime}+\mathrm{X}^{\prime} \mathrm{YZ}$ \\
\hline Q10 & $X^{\prime} Y^{\prime}+Y^{\prime} Z^{\prime}+Z^{\prime} X^{\prime}+X Y Z$ \\
\hline Q11 & $X^{\prime} Y^{\prime} Z^{\prime}+X^{\prime} Y Z+X Y^{\prime} Z+X Y Z^{\prime}$ \\
\hline Q12 & $X^{\prime} Y^{\prime} Z+X^{\prime} Y Z^{\prime}+X Y^{\prime} Z^{\prime}+X Y Z$ \\
\hline Q13 & $X^{\prime} Y^{\prime} Z+X^{\prime} Y Z^{\prime}+X Y^{\prime} Z^{\prime}$ \\
\hline Q14 & $\mathrm{XY}+\mathrm{YZ}+\mathrm{ZX}+\mathrm{X}^{\prime} \mathrm{Y}^{\prime} \mathrm{Z}^{\prime}$ \\
\hline
\end{tabular}

Table III: two variables Symmetric Function

\begin{tabular}{|l|l|}
\hline P1 & $\mathrm{X}+\mathrm{Y}$ \\
\hline P2 & $\mathrm{XY}$ \\
\hline P3 & $\mathrm{X}^{\prime} \mathrm{Y}^{\prime}$ \\
\hline P4 & $\mathrm{X}^{\prime}+\mathrm{Y}^{\prime}$ \\
\hline P5 & $\mathrm{XY}+\mathrm{X}^{\prime} \mathrm{Y}^{\prime}$ \\
\hline P6 & $\mathrm{X}^{\prime} \mathrm{Y}+\mathrm{X} \mathrm{Y}^{\prime}$ \\
\hline
\end{tabular}


The 2-variable and 3-variable symmetric function Implementation using RUG is given in the Fig.4.

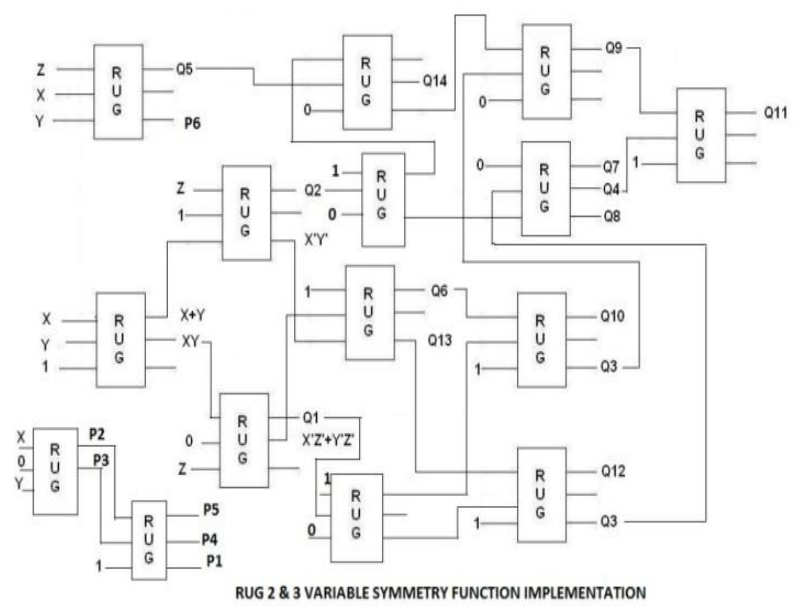

FIG.4: 2-variable and 3-variable symmetric function Implementation using RUG

The Fig.5. Shows effectiveness of RUG while comparing with the other reversible gates in implementing the two and three variable symmetric function implementation.

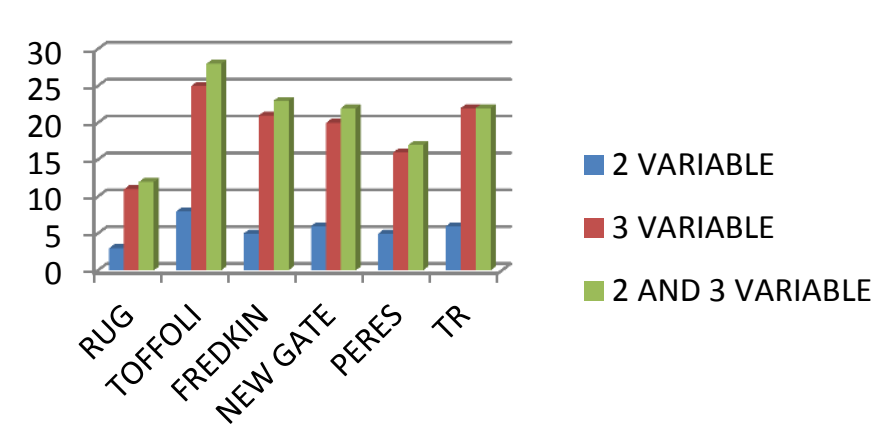

FIG.5: Number of gates taken (y-coordinate) vs RUG, TOFFOLI, FREDKIN, NEW GATE, PERES and TR Gates (x-coordinate).

Now it can be easily seen form FIG. 3 and FIG. 5 that the RUG implements all 13 standard function and all 2variable,3-variable and $2 \& 3$-variable symmetric function in less number of gates and garbage outputs. So RUG is very efficient for circuit designing while taking energy saving into consideration.

\section{Conclusion}

This work introduces effectiveness of Reversible Universal Gate (RUG) based on QCA logic gate structure referred to as the RUG which satisfies the role of universal reversible gate in an energy efficient logic design. It is established that the RUG based QCA circuits are cost-effective in terms of number of logic gates, garbage outputs as compared to the designs based on existing reversible gates. This cost effective feature of RUG, with high device density, will play important role in CMPs with multi-processors. Realization of symmetric functions and 13 standard function using RUG has been reported. It establishes the fact that any reversible gate with universal, majority and XOR function can enable low power QCA designs.

\section{References}

[1] C.S.Lent and P.D.Tougaw, "A Device Architecture for Computing with Quantum Dots," Proc.IEEE,vol.85,no.4,pp.541-577,Apr.1997.

[2] Momenzadeh, M, Jing Huang, Tahoori, M.B., Lombardi,F. ,'Characterization, test, and logic synthesis of and-or-inverter (AOI) gate design for QCA implementation', in IEEE Transaction on ComputerAided Design of Integrated Circuits and Systems, Vol. 24, pp-1881-1893 No. 12, December,2005.

[3] R. Landauer, 'Irreversibility and Heat Generation in the Computational Process' , IBM Journal of Research and Development, 5, pp. 183-191, 1961.

[4] C.H. Bennett, 'Logical Reversibility of Computation' ,IBM J.Research and Development, pp. 525-532, November 1973.

[5] J. Timler and C. Lent, 'Maxwells demon and quantum dot cellular automata', Journal of Applied Physics, vol.94, no. 2,pp. 1050, 2003.

[6] Bibhash Sen, Divyam Saran, Mousumi Saha and B K Sikdar, "Synthesis Of Reversible Universal Logic Around QCA With Online Testability", in Proceedings of $2^{\text {nd }}$ IEEE Conference ISED'11, Page(s): 236 - 241 Kochi,India, 2011.

[7] T. Toffoli, 'Reversible computing',MIT, Cambridge, MA,1980. Tech. Rep. MIT/LCS/TM-151.

[8] E. Fredkin and T. Toffoli, 'Conservative logic', Int. J. Theor.Phys.,vol. 21, no. 3/4, pp. 219253, 1982.

[9] H. Rahman. D.K. Das and B.B. Bhattacharya,'Mapping symmetric functions to hierarchical modules for pathdelay fault testability', Proceedings, Asian Test Symposium (ATS), IEEE CS Press, USA, pp. 284-289, Nov. 2003.

[10] Yinshui Xia, Keming Qui, "Design and Application of Universal Logic Gate Based on Quantum-Dot Cellular Automata", pp.335-338,2008. 